\title{
Computational study of electron transport in [FeFe] hydrogenase enzymes
}

\author{
Rakesh C Puthenkalathil* and Bernd Ensing \\ Van 't Hoff Institute for Molecular Sciences (HIMS), University of Amsterdam, Science \\ Park 904, 1098 XH Amsterdam, The Netherlands \\ E-mail: rakeshudayam@gmail.com
}

\begin{abstract}
$[\mathrm{FeFe}]$ hydrogenase enzymes can reversibly catalyze the conversion of protons into molecular hydrogen. The active site of the $[\mathrm{FeFe}]$ hydrogenase enzyme is buried inside the protein. The transport of electrons and protons to the active site of the protein is crucial for an efficient catalytic cycle. A chain of iron-sulfur cubane cofactors forms a pathway for the electron transfer in these $[\mathrm{FeFe}]$ hydrogenases. We have studied the electron transfer process via the iron-sulfur clusters in the enzyme using classical molecular dynamics simulations. Our simulations show that the protein matrix acts as a porous medium for the transport of water molecules in and out during the electron transfer process. When an electron is transferred through the pathway, solvent water molecules penetrate the protein, forming hydrogen bonded networks and hydrating the electron accepting cubane clusters. The reorganization of the protein and the penetrating water molecules have a large effect on the free energy landscape of the electron transfer, via the formation of favorable hydrogen bonds with the reduced ironsulfur cluster, thereby stabilizing the electron at the cofactors.
\end{abstract}




\section{Introduction}

Electron and proton transfer reactions are fundamental steps in many biological systems. ${ }^{1,2}$ In various redox active enzymes, such as ubiquinone oxidoreductase ${ }^{3}$ and hydrogenases, ${ }^{4}$ a chain of iron-sulfur $(\mathrm{FeS})$ clusters enables the transfer of electrons to the active site. Di-iron hydrogenase is a redox active enzyme that converts protons into molecular hydrogen. The active site of the $[\mathrm{FeFe}]$ hydrogenase, commonly known as the "H-cluster", is buried inside the protein matrix. The transfer of protons and electrons from the surface of the protein to the H-cluster plays a vital role in the efficiency of the enzyme. ${ }^{5}$ Fluctuations in the protein scaffold are thought to be essential for creating favorable electrostatic interactions that enable efficient charge transport in these enzymes. ${ }^{6}$ However, the mechanistic details of the electron transport and the role of the protein fluctuations in this process are still largely unknown.

The $[\mathrm{FeFe}]$ hydrogenase protein covalently binds one $\mathrm{Fe}_{2} \mathrm{~S}_{2}$ sub cluster (FS2) and four $\mathrm{Fe}_{4} \mathrm{~S}_{4}$ cubane clusters, labeled FS4A, FS4B, FS4C, and FS4D in Figure 1, that can accept and donate electrons. The FS4A cluster is closest to the catalytically active di-iron site (2Fe), and considered as a part of the H-cluster. The Fe atoms of the clusters are attached to the protein via cysteine ligands, except the cubane cluster located closest to the solvent accessible protein surface (FS4D), which is bound by three cysteines and one histidine ligand to the protein. A previous computational study has shown that the reduction of the FS4A cluster causes the formation of a water channel in the protein towards the reduced cubane cluster. ${ }^{7}$ Also in studies of other redox-active protein systems, such as the respiratory complex I, dynamic water chains were found to couple to the electron transfer process. ${ }^{8,9}$

Previously the electron transfer process along the FeS clusters was studied using molecular dynamics simulations. ${ }^{7}$ The study found that the electron transport is a downhill process, and by partitioning the protein into seven coarse-grained sites, they could analyze the contributions of the protein and the solvent to the transport free energy. 

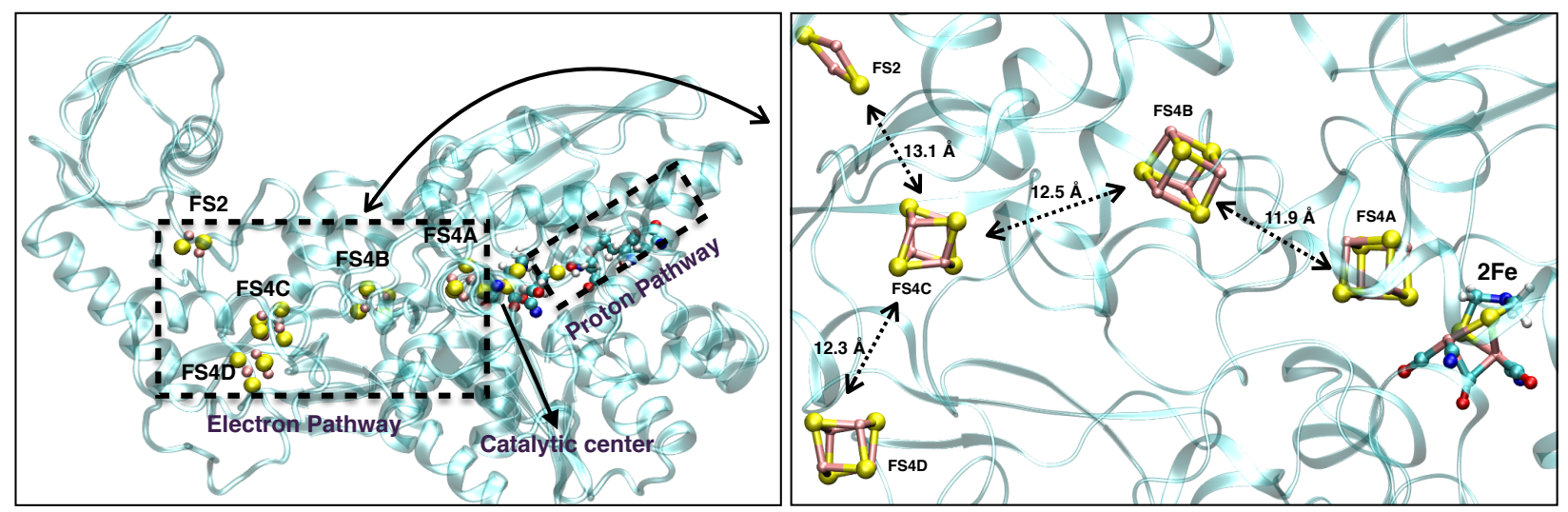

Figure 1: (Left) The structure of the [FeFe] hydrogenase enzyme, showing the catalytic Hcluster at the center, the electron transporting cubane clusters at the left, and the proton transfer channel on the right. (Right) Close-up of the electron transfer chain of iron-sulfur cofactors. The average center of mass distance between the cubane clusters is indicated. The cubane clusters are labeled FS4A to FS4D, and on the top-left is the FS2 cluster. 2Fe is the active site where proton reduction and $\mathrm{H}_{2}$ formation takes place.

In this study, we perform molecular dynamics (MD) simulations of the solvated protein at different oxidation states of the $\mathrm{FeS}$ clusters. In particular, we investigate the protein and solvent reorganisation upon reducing one FeS cluster with respect to the reference state of the protein in which all clusters are in the oxidized form. Even though both FS4D and FS2 clusters are located close to the surface of the enzyme, the FS4D cluster is considered as the electron receiving moiety from a Ferrodoxin donor. ${ }^{10}$ The role of the FS2 cluster in the electron transport is still not well understood. It is proposed that the role of FS2 is to fine-tune the pathway by electronic interaction with other the clusters. ${ }^{10}$ Using the free energy perturbation technique, we compute the free energy profiles for the electron transfer from one cubane cluster to the next, that is for the three transfer reactions: FS4D $\rightarrow \mathrm{FS} 4 \mathrm{C}$, $\mathrm{FS} 4 \mathrm{C} \rightarrow \mathrm{FS} 4 \mathrm{~B}$, and $\mathrm{FS} 4 \mathrm{~B} \rightarrow \mathrm{FS} 4 \mathrm{~A}$.

The rest of this chapter is organized as follows, the methods section describes the details of our classical MD simulations and a recapitulates Marcus' theory of electron transfer. In the results section, we first analyse the water density to investigate the protein reorganisation and the solvent water penetration upon FeS reduction, as well as the stability of the hydrogen 
bond networks and the formation/breaking of the hydrogen bonds with the FeS cluster along the electron transfer pathway. Next, we analyse the effect of fractional charge transfer on the formation of the water network. The final part is dedicated to the electron transfer free energy profiles computed using Marcus theory. Three free energy profiles are calculated and discussed, which corresponds to the three electron transfer reactions between the cubane clusters, from the protein surface to the catalytic site. We end with conclusions.

\section{Methods}

The initial atomistic structure of the [FeFe] hydrogenase for our simulations is based on crystal structure PDB:3C8Y of the enzyme from the protein databank. ${ }^{11}$ Molecular dynamics simulations are carried out using the Gromacs simulation package. ${ }^{12}$ The CHARMM27 forcefield with CMAP corrections is used to model the protein. ${ }^{13,14}$ For the FeS clusters, we use the parameters devised by Chang and $\mathrm{Kim}^{15}$ and further modified by McCullagh and Voth. ${ }^{7}$ The TIP3P water model is used for the solvent. ${ }^{16}$ The protein is solvated with 30,000 water molecules and sodium and chlorine ions to create a $0.1 \mathrm{M} \mathrm{NaCl}$ solution, thereby neutralizing the protein charge. After an initial energy minimization, the system is equilibriated for $10 \mathrm{~ns}$ in the NPT ensemble to a temperature of $T=300 \mathrm{~K}$ and a pressure of $p=1 \mathrm{~atm}$. A subsequent production run of $120 \mathrm{~ns}$ is carried out in the NVT ensemble for analysis. All simulations are performed with the default leap-frog integration scheme and a time step of $2 \mathrm{fs}$, using $\operatorname{LINCS}^{17}$ to constrain all bonds. The v-rescale thermostat ${ }^{18}$ and the Parrinello-Rahman barostat ${ }^{19}$ are used to sample an isothermal-isobaric ensemble.

Free energy profiles for the electron transfer processes are calculated using free energy perturbation. ${ }^{20}$ We compute these Marcus plots for the transfer of one electron from one FeS cubane cluster to the next, starting at the FS4D cluster, which is nearest to the protein surface, and ending at FS4A, which is located at the catalytic reaction center. The free 
energy can be calculated as a function of the vertical energy gap, $\Delta E$ :

$$
\Delta F(\Delta E)=-k_{B} T \ln [P(\Delta E]
$$

in which $k_{B}$ is the Boltzmann constant and $T$ is the absolute temperature. $\Delta E$ is defined as the energy required to transfer an electron from a reduced complex to an oxided complex in a given nuclear configuration. The vertical energy gap distribution is calculated in two different equilibrium MD simulations, one in the reactant state of the system, in which $\Delta E$ is evaluated for the forward electron transfer reaction, and the other in the product state of the system, in which $\Delta E$ is evaluated for the backward reaction. The $\Delta E$ is calculated at intervals of $50 \mathrm{ps}$ during these simulations. The computed $\Delta E$ histograms are fitted with a Gaussian function, after which the parabolic free energy curves are obtained with Eq. 1. We have used two different approaches to compute $\Delta E$. In the first approach, we employ for the reduced cubane the forcefield parameters from the paper by McCullagh and Voth. ${ }^{7}$ In the second approach, we use for the reduced cubane only the charges from that paper, while leaving the other forcefield parameters as in the oxidized state of the protein. The first approach resulted in very large, unrealistic, $\Delta E$ values, which we attribute to the rather stiff and different parameters for the cubane cofactors. In the second approach, we obtain much better results, comparable with experimental observations. Note that in this second approach, the inner sphere reorganization may thus be underestimated. However, since the inner sphere reorganization is expected to be the same for all cubane cofactors, we can assume that these inner sphere contributions approximately cancel out for the charge transfer from one cubane to another. In this work, we only present the results from the second approach.

Figure 2 shows an illustrative free energy plot, in which the profiles for the reactant and the product states are obtained from the energy gap distributions using Eq. $1 .{ }^{21-24}$ When the 
linear response approximation (of the molecular environment polarization upon a transfer of charge) underlying Marcus theory holds, the two curves are parabolas with the same curvature. Moreover, the reaction free energy, $\Delta F$, and the reorganization free energy, $\lambda$, can then be calculated using equation 2 and equation 3.

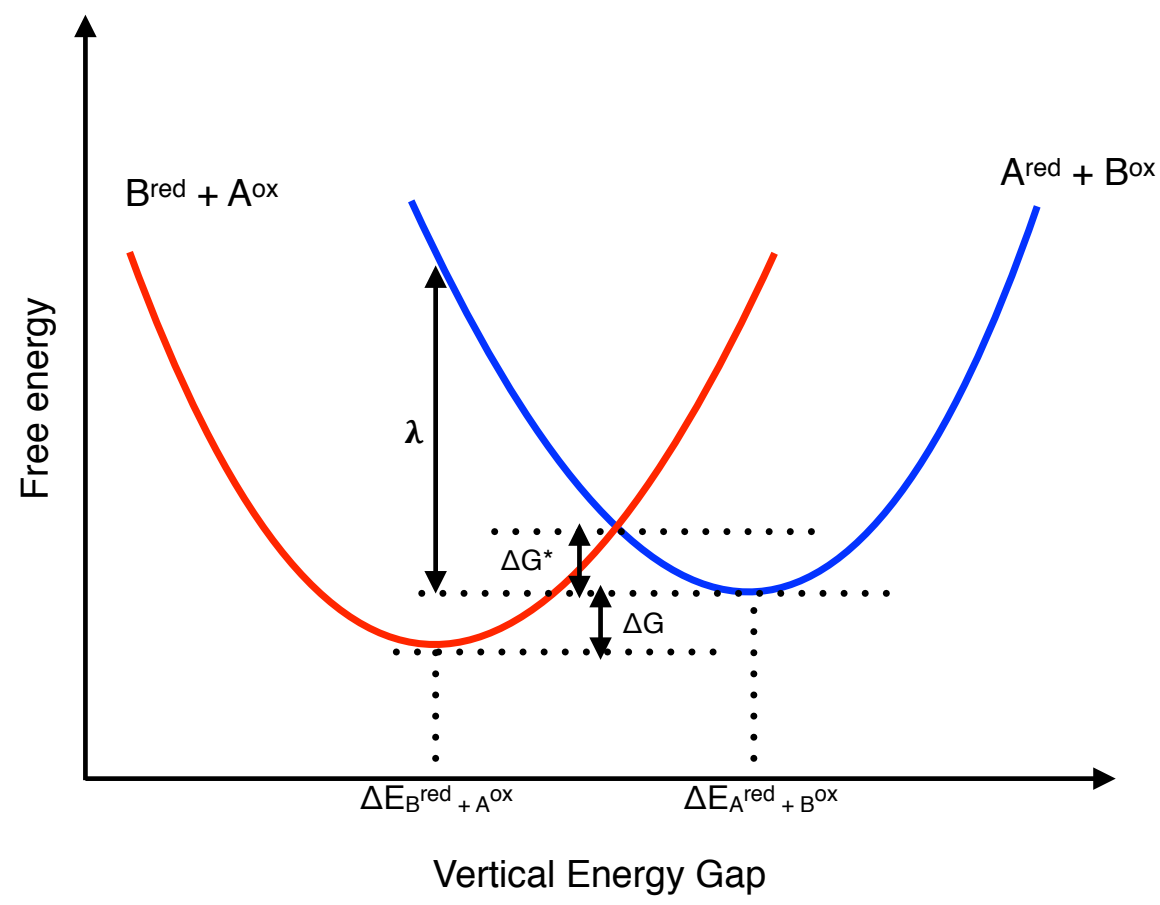

Figure 2: Illustrative Marcus plot showing the two parabolic free energy curves as a function of $\Delta E$ for the electron transfer between two redox compounds $\mathrm{A}$ and $\mathrm{B}$.

$$
\begin{gathered}
\Delta F=\frac{1}{2}\left(\langle\Delta E\rangle_{P}+\langle\Delta E\rangle_{R}\right) \\
\lambda=\frac{1}{2}\left(\langle\Delta E\rangle_{P}-\langle\Delta E\rangle_{R}\right)
\end{gathered}
$$

in which $\langle\Delta E\rangle_{R}$ and $\langle\Delta E\rangle_{P}$ are the average energy gaps in the reactant and product states, respectively. From the reaction free energy and the reorganization free energy, the activation free energy of the electron transfer can be calculated as: 


$$
\Delta F^{*}=\frac{(\lambda+\Delta F)^{2}}{4 \lambda}
$$

\section{Results and Discussion}

A series of simulations is performed of the solvated di-iron hydrogenase, in which every time a different iron-sulfur cluster is in its electronically reduced state, while all other clusters are in the oxidized form. We also ran a simulation of the system with all the clusters in the oxidized form, which we denote $\mathrm{FS}^{\text {all-ox }}$. The clusters are labeled as in Figure 1. FS2 $2^{\text {red }}$ signifies that the $\mathrm{Fe}_{2} \mathrm{~S}_{2}$ cluster is in its reduced form and the other clusters are in their oxidized form. Similarly FS4A ${ }^{\text {red }}$, FS4B ${ }^{\text {red }}$, FS4C ${ }^{\text {red }}$, and FS4D ${ }^{\text {red }}$ indicates the respective cubane cluster $(\mathrm{A}, \mathrm{B}, \mathrm{C}$, or $\mathrm{D})$ that is in its reduced state and the other clusters are in the oxidized state.

\section{Solvent water permeation into the electron transfer pathway}

To investigate the possible permeation of solvent water into the hydrogenase protein as a function of the cubane oxidation state, average water density maps are computed from the $100 \mathrm{~ns}$ MD trajectories using the VolMap tool that is implemented in the VMD software. ${ }^{25}$

The results are shown in Figure 3. The average density of water molecules within a $10 \AA$ radius of the FeS clusters is calculated.

In the oxidized form of the cubane clusters, no water channels close to the cubane clusters are observed (Figure 3(a)). The water density map only shows some crystallographic water in specific pockets of the enzyme. Figure 3(b) shows the water density at the first reduced state, FS2 ${ }^{\text {red }}$, in which the FS2 cluster has an extra electron. As in the case of the fully oxidized cluster, water channels are absent in the protein in the FS2 ${ }^{\text {red }}$ state, although some changes in the water density are observed. The remaining panels, c to f, in Figure 3 show 


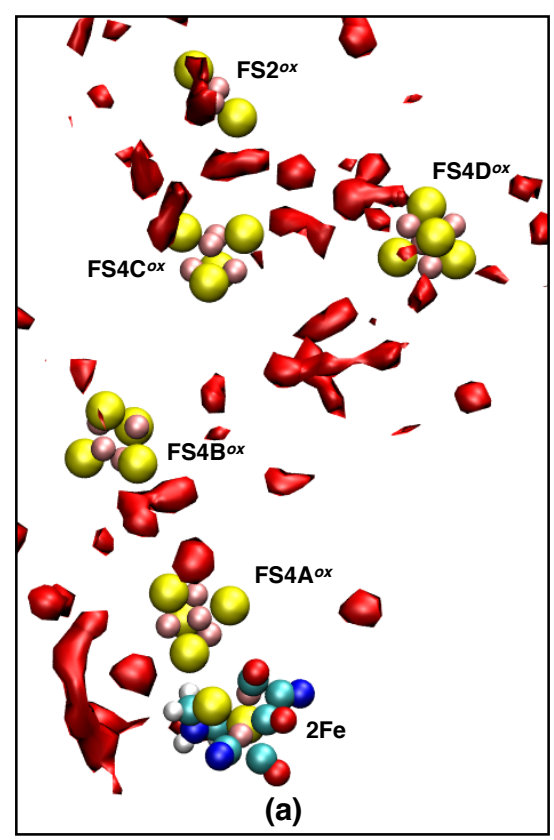

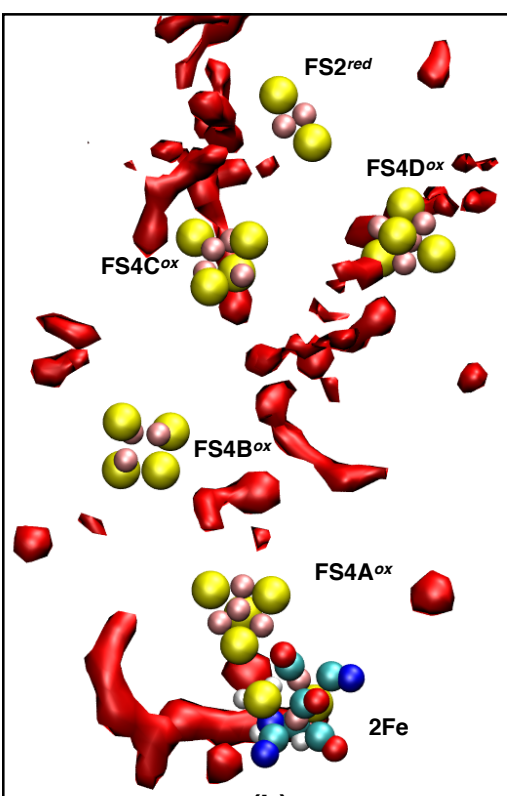

(b)

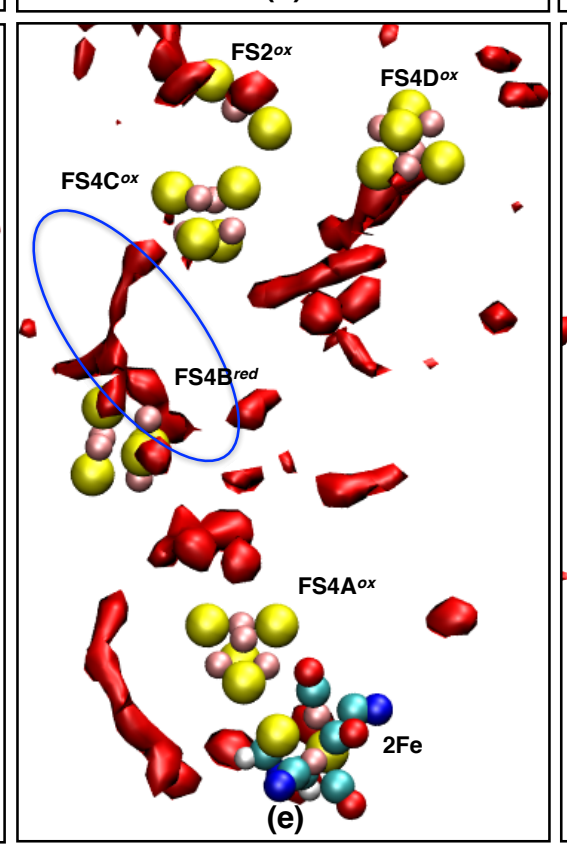

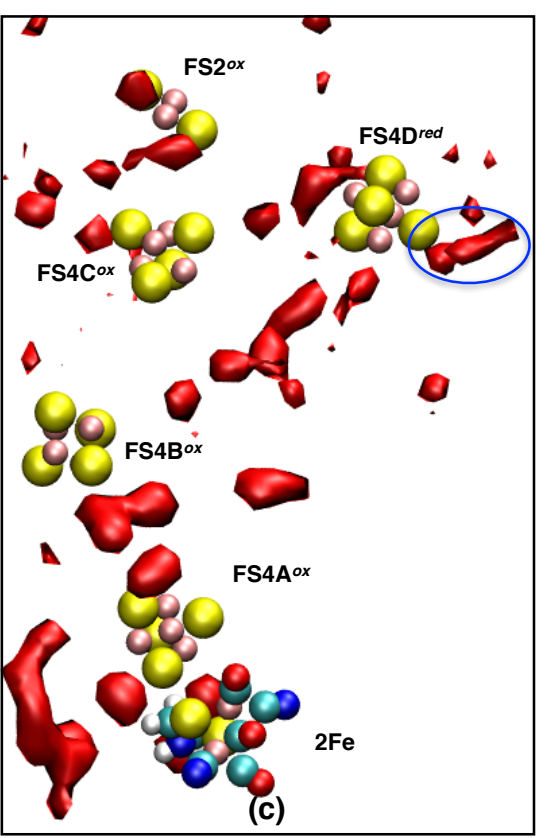

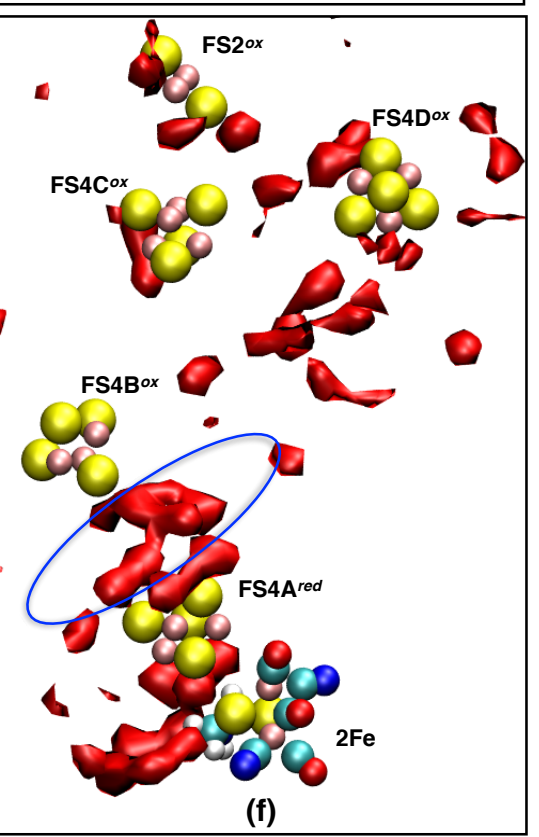

Figure 3: Water density maps showing the penetration of solvent water into the protein matrix in the vicinity of the cubane clusters for the different oxidation states. Only iron (pink) and sulfur (yellow) atoms are shown, and the catalytic 2Fe cluster at the bottom. Red isosurfaces show the average density of water molecules at an isovalue of 0.05 . Water channels to the FeS clusters are marked in blue elipses. Panel a: FS ${ }^{\text {all-ox }}$, b: FS2 ${ }^{\text {red }}$, c: FS4D ${ }^{\text {red }}$, d: FS4C ${ }^{\text {red }}$, e: FS4B ${ }^{\text {red }}$, f: FS4A ${ }^{\text {red }}$. 
that in each case a visible water channels is formed from the solvent at the protein surface to the reduced cubane cluster (indicated by the blue ovals). These six MD simulations were carried out consecutively, restarting the next run from the last frame of the previous run. This allows us to observe that the shown water channels appear relatively quickly, within 15-20 ns, then remain stable throughout the rest of the simulation, and disappear within 25 ns after switching to the next oxidation state. These water density maps show that there is a considerable reorganization of the protein structure that allows water molecules to penetrate and form channels after electron reduction of any of the $\mathrm{F}_{4} \mathrm{~S}_{4}$ clusters. Moreover, the simulations show that these changes in permeability and water diffusion happen on the nanosecond time scale. We note however that, although water channels are not observed in the vicinity of the oxidized cubane clusters, nevertheless during electron transport the water penetration is expected to be concerted with the charge transfer (instead of occurring after the charge transfer) to stabilize a hydrated reduced product state.

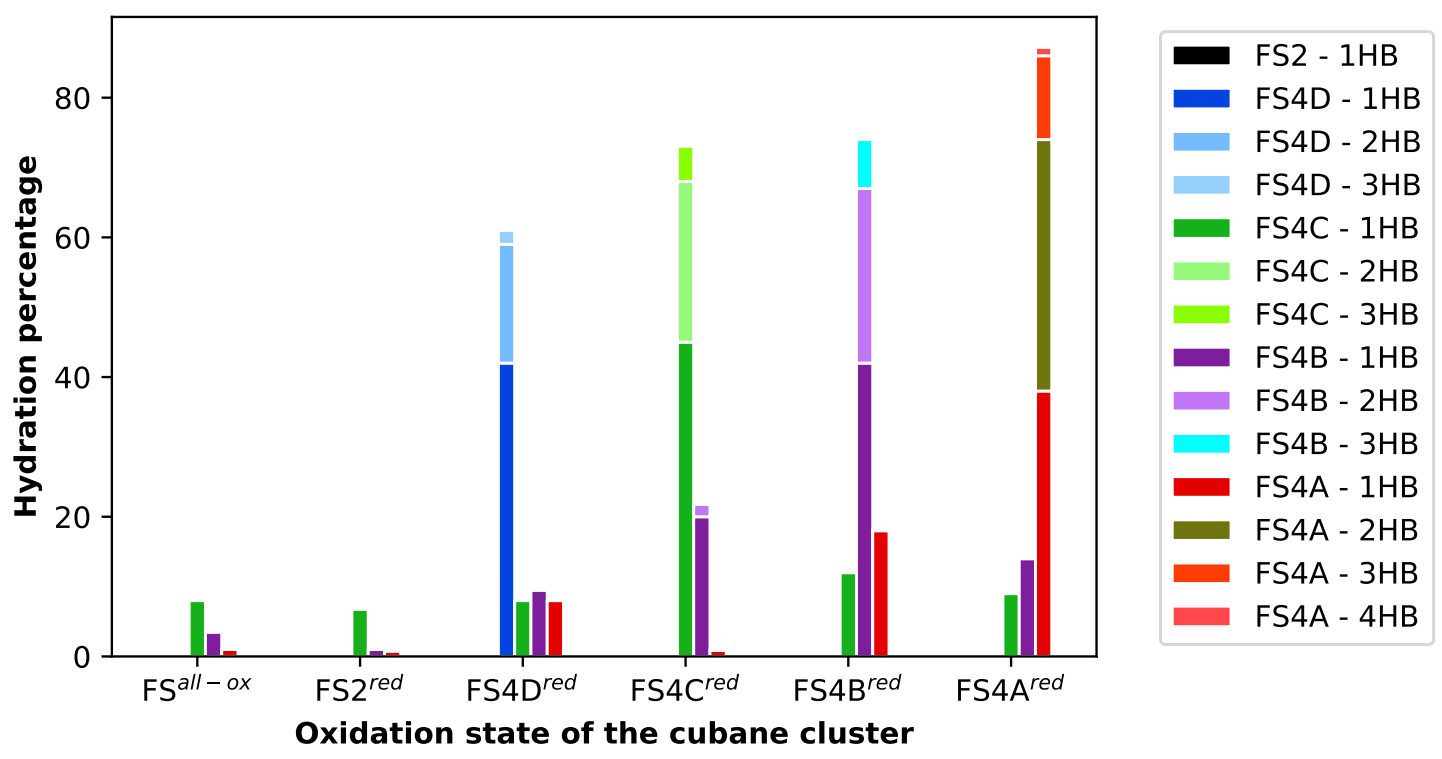

Figure 4: Average number of water molecules donating hydrogen bonds to each FeS cluster for the six different oxidation states. The reduced cluster is denoted on the x-axis. The color bar shows the number of $\mathrm{H}$-bonds to each cluster. Note the increase in H-bond donation at the cluster that accepts an electron. 
To quantify the amount of hydration of the reduced cubane clusters by penetrating solvent water molecules, we compute the hydrogen bond population between water and the $\mathrm{Fe}_{4} \mathrm{~S}_{4}$ clusters. Here, a hydrogen bond is counted when the distance between a water oxygen and a cubane sulfur atom is less than $3.0 \AA$ and the hydrogen bond angle is larger than 150. The results, shown as a histogram for each oxidation state in Figure 4, confirm the previous observations from the water density maps. In the oxidized state and in the FS2 ${ }^{\text {red }}$ state, no significant hydration of any of the cubane clusters is seen, apart from some minimal water interaction with the FS4C cluster during less than $10 \%$ of the time. Instead, when any of the cubane clusters are reduced, significant hydration is observed by two to three water molecules at the reduced cluster. Note also that the total time percentage of H-bond forming is increasing from the outermost FS4D cubane (ca. 60\%) to the innermost FS4A cluster (more than 80\%). Simultaneously, some minor hydration is observed at the neighboring clusters, which are in the oxidized state.

Previously, redox anti-cooperativity of the FS4A cluster with the nearby FS4B cluster has been studied, ${ }^{26}$ which suggested that a reduced FS4B cluster causes the FS4A cluster to be reduced at more negative reduction potentials due to the repulsive interaction between the reduced clusters. ${ }^{26}$ Interestingly, our hydrogen bond analysis shows also another, cooperative, effect. In particular, we observe that reduction of FS4B results in a somewhat increased hydration at the neighboring FS4A cluster (see the red bar at FS4B ${ }^{\text {red }}$ in Figure 4). Moreover, a similar cooperative interaction can be observed for the electron transfer from FS4C to FS4B; in the FS4C $\mathrm{C}^{\text {red }}$ state, already a significant increase upto $20 \%$ in the H-bond population is seen at the (still oxidized) FS4B cluster (see the purple bar). Hydrogen bond donation by water results in a positive electrostatic potential at the accepting cubane cluster, thus effectively lowering the reduction potential and promoting the electron transfer.

Cooperative interaction between the clusters is important for the electron transfer process. Previous work has shown that structural water molecules increase the rate of electron 


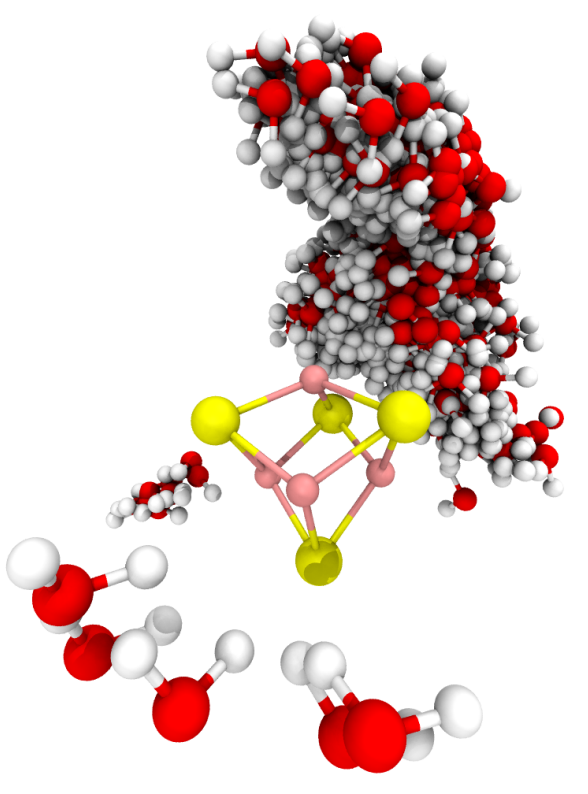

(a)

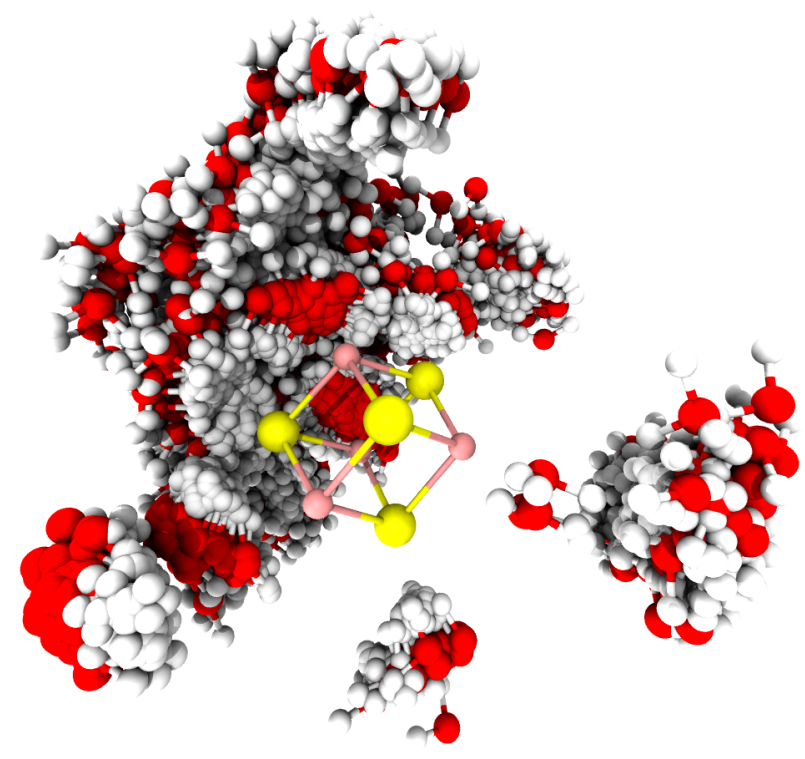

(b)

Figure 5: Superposition of water molecule configurations within $5 \AA$ of cubane cluster FS4A, taken from $100 \mathrm{~ns}$ trajectories at intervals of 0.5 ns. (a): oxidized state; (b): reduced state. Only a single configuration of the $\mathrm{Fe}_{4} \mathrm{~S}_{4}$ cluster is shown.

transfer in biological systems. ${ }^{27,28}$ Studies on the respiratory complex I and NADH:ubiquinone oxidoreductase, which contains the same FeS cofactors, have shown that the protein environment acts as a porous medium, which allows the water molecules to diffuse in and out during the electron transfer process. ${ }^{3,29,30}$ Here, we see that the [FeFe] hydrogenase enzyme behaves in a similar manner, which suggests that the formation of water channels may be a common feature among the class of redox proteins that contains FeS clusters for electron transfer.

To provide a molecular picture of the change in hydration around the cubane clusters, Figure 5 shows a superposition of 200 configurations of the nearby water molecules taken from the $100 \mathrm{~ns}$ MD trajectories at $0.5 \mathrm{~ns}$ intervals. Figure 5a shows the water molecules near the FS4A cluster in the oxidized state and Figure 5b shows this in the reduced state. In the oxidized state, we see configurations from a single water molecule on one side of the 


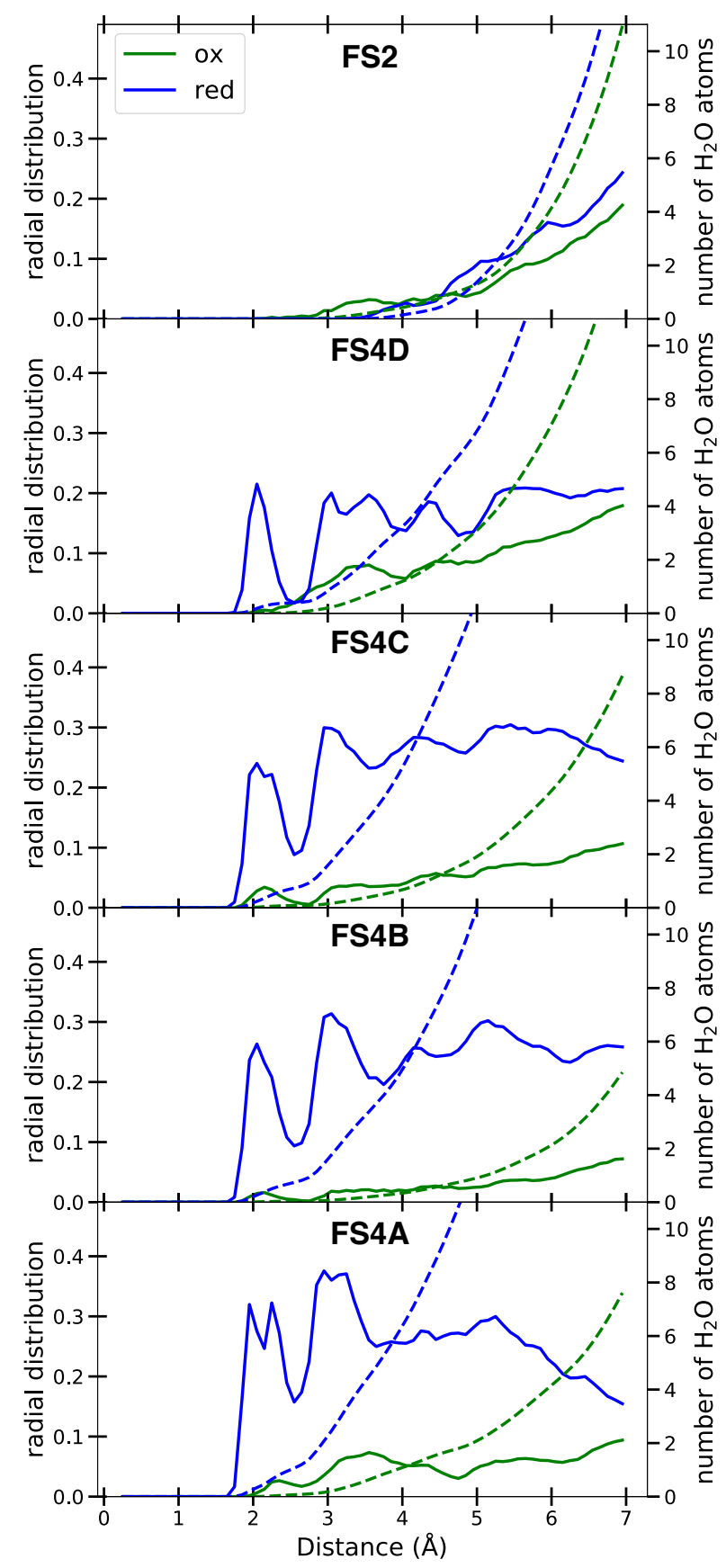

Figure 6: Radial distribution functions of water $\mathrm{O}$ and $\mathrm{H}$ atoms around the cubane $\mathrm{Fe}$ and $\mathrm{S}$ atoms in the oxidized (solid green lines) and reduced (solid blue lines) states. Cumulative coordination numbers (dashed lines) correspond with the vertical axes on the right side.

cubane that is rather mobile and moving in random orientations. Instead, in the reduced state, the density of water molecules is significantly higher, with several water clusters due 
to water molecules that are strongly interacting by donating a hydrogen (white) bond to cubane sulfer atoms (yellow) or coordinating with the oxygen (red) lone pairs to iron atoms (pink).

The difference in water coordination is further quantified by the radial distribution function shown in Figure 6. Here, the solid curves show the radial distribution of water oxygen and hydrogen atoms around the cubane iron and sulfur atoms in the oxidized (green) and reduced (blue) states for all FeS clusters. The dashed lines show the radial integration of these pair-correlation functions, resulting in the cumulative coordination numbers shown on the right-hand-side axes. In the oxidized state, the water density in the neighborhoods of the FeS clusters is very low. The integrated density is at most 3 atoms (i.e. $1 \mathrm{H}_{2} \mathrm{O}$ ) within spheres of $6.5 \AA$ radius from the Fe and S atoms. Instead, when a cubane cluster accepts an electron, a clear structure of coordination shells appears, with a first peak around $2 \AA$ indicating the Fe-O and $\mathrm{S}-\mathrm{H}$ interactions. Note that the number of water molecules is somewhat less around FS4D ${ }^{\text {red }}$ compared to the other cubane clusters, and that hardly any hydrating water structure is seen around the FS2 cluster, even when it is reduced (top panel).

Also from the outside, the porous nature of the protein scaffold can be visualized, for example showing the opening of new water channels in the reduced state of the FS4A cluster. Figure 7 shows the protein surface in the oxidized state (left) and a reduced (right) state, in which we depict several water channels that open up in the reduced state of the FS4A cluster.

\section{Cubane hydration at partial charge transfer}

In Marcus' theory of electron transfer, the response of the polar medium, measured by the gap energy, $\Delta E$, needed to transfer some charge, is assumed to be proportional with the amount of charge that is transferred. To test this assumption, we would like to vary the 


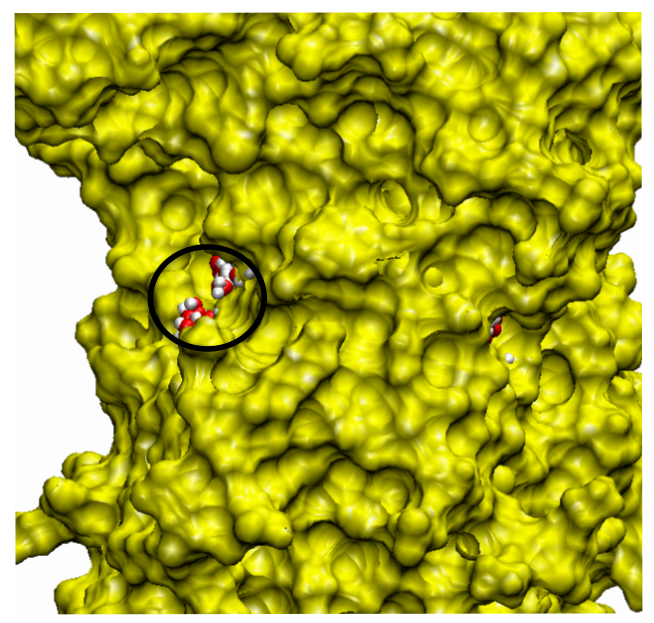

(a)

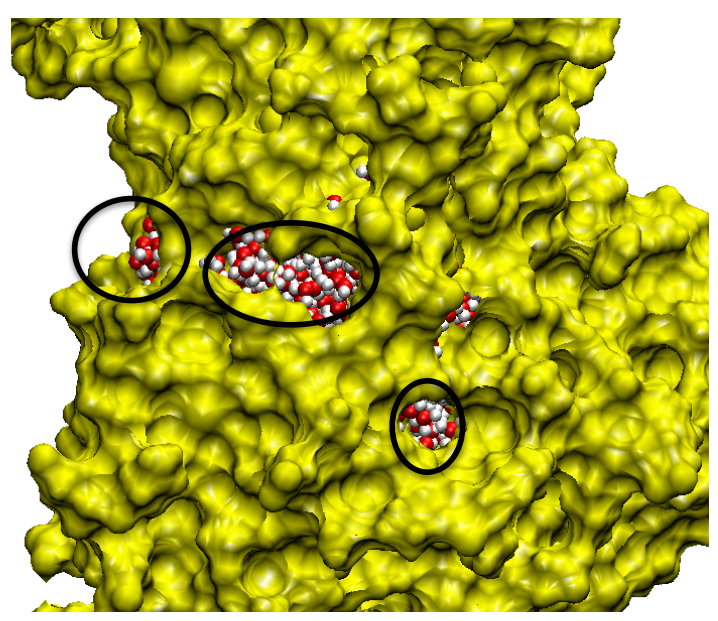

(b)

Figure 7: Superposition of water molecules that are within $10 \AA$ of the FS4A cluster protruding at the protein surface. The water configurations are taken from a 100 ns trajectory of the FS $\mathrm{S}^{\text {all-ox }}$ state (left panel) and of the FS4A ${ }^{\text {red }}$ state (right panel) at intervals of $0.5 \mathrm{ns.} \mathrm{A}$ single frame of the protein surface is shown. Black circles show the water networks connecting the FS4A cluster and the outside solvent.

amount of charge that is transferred, and monitor the amount of molecular outer sphere reorganization. This would be very difficult or impossible to do in an experimental setup, however, in a molecular simulation, it is not a problem to transfer amounts of charge that are only a fraction of an electron charge. To this end, we set up five simulations with partial charges on the cubane clusters. The atomic charges, $q$, on the clusters are calculated according to the equation: $q=(1-x) \cdot q\left[\mathrm{FS}_{\mathrm{B}} \mathrm{B}^{\mathrm{red}}\right]+x \cdot q\left[\mathrm{FS}^{\mathrm{A}} \mathrm{A}^{\mathrm{red}}\right]$. At $x=0$, FS4B is in its fully reduced form and FS4A is in the oxidized form. The $x$ values taken for the simulations are $0,0.3,0.5,0.7$ and 1 . At $x=1$, the electron is completely transferred to the FS4A cluster. Similarly, five simulations are carried out to model the partial charge transfer between the FS4C and FS4B clusters. The force field parameters (other than the charges) are taken to be those of the oxidized protein for all the simulations.

In Figure 8, the hydrogen bond population due to hydrating water molecules at the FeS clusters is shown for the two partial charge transfer reactions, FS4B $\rightarrow$ FS4A (left panel) and FS4C $\rightarrow$ FS4B (right panel). As expected, the hydrogen bond population decreases on the 


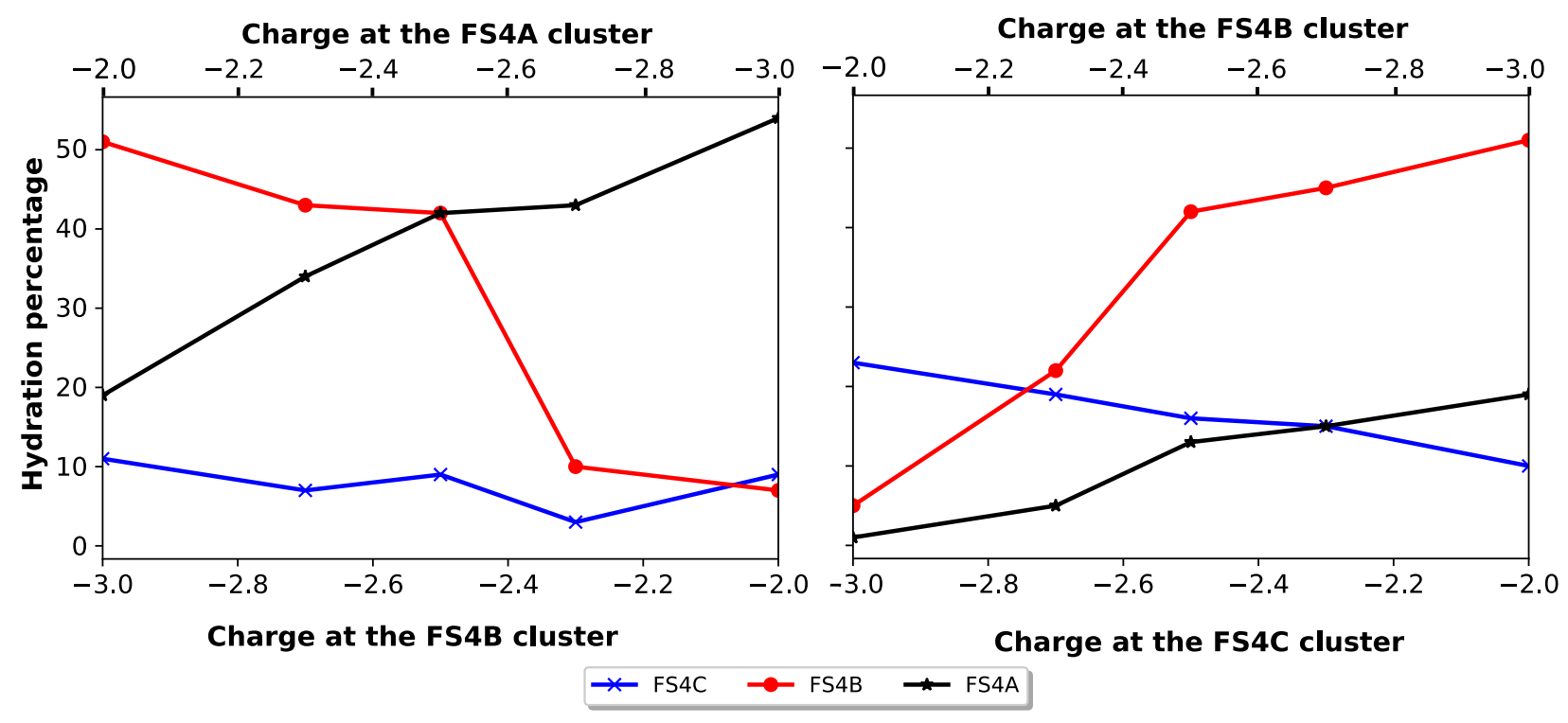

Figure 8: Average numbers of hydrogen bonds donated by hydrating water molecules to the FS4A, FS4B, and FS4C cubane clusters during partial charge transfer in steps of $0,0.3,0.5$, 0.7, and 1.0 transferred electron. Left panel: partial electron transfer from FS4B to FS4A. Right panel: partial electron transfer from FS4C to FS4B.

electron donating cluster and increases on the accepting cluster, as the amount of transferred charge increases. Note however that the change in H-bond population does not follow a linear trend with the amount of charge transferred. The change in the H-bond population at a cluster is large when the oxidized cluster (with a formal charge of -2) receives a small amount of negative charge, but the change is significantly smaller when the reduced cluster (with a formal charge of -3) donates a small amount of charge. The graphs in Figure 8 seem to imply two regimes: for the first half of the electron transfer when the oxidized cluster accepts up to half an electron, the slope is rather steep; this is followed by the second half in which the slope is more flat. This non-linear behavior is perhaps not surprising for this solvent permeation and cluster hydration, as it is reminiscent of wetting behavior in confined spaces, for example seen in experiments in which the separation between two dissolved surfaces is varied, showing hysteresis upon increasing and decreasing of the separation. Unfortunately, such hysteresis leads to difficulties in sampling, so that probably longer simulations and more 
partial charge values should be computed to quantify more accurately the non-linear solvent reorganization observed here.

\section{Free energy of electron transfer between the cubane clusters}

The internal transport of electrons from the protein exterior to the catalytic center is believed to take place via three successive electron transfer jumps: from FS4D to FS4C, from FS4C to FS4B, and from FS4B to FS4A. As already indicated in Figure 1, the distances between the

consecutive cubane clusters are larger than $10 \AA$. However, electrons are known to traverse by quantum tunneling over distances up to $14 \AA$ in biological systems. ${ }^{31}$ Electron transfer is predominantly non-adiabatic (aka diabatic) if the distance between the donor and the acceptor is more than $10 \AA .{ }^{22}$ In Figure 9, we show the instantaneous distances between the centers of mass of the four cubane clusters, in the different oxidation states of the protein. Clearly, the electron transfer cofactors are arranged in the [FeFe] hydrogenase enzyme with distances, $r$, that fluctuate within $11 \leq r \leq 14 \AA$. From the analysis of the electron donor-acceptor distances, we thus conclude that in hydrogenase the electron transfer can be considered a diabatic transfer.

The free energy profiles of the three electron transfer steps are shown in Figure 10, starting with that of the electron transfer from FS4D to FS4C on the left (panel a). Note that the blue free energy curve on the right side is from the reactant $(\mathrm{R})$ state, FS4D ${ }^{\text {red }}+\mathrm{FS}_{4} \mathrm{C}^{\text {ox }}$, and the orange curve of the product $(\mathrm{P})$ state, FS4D ${ }^{\mathrm{ox}}+\mathrm{FS}_{4} \mathrm{C}^{\mathrm{red}}$, is on the left side. The zero of the free energy scale is set to the minimum of the reactant curve. This first electron transfer is exergonic by $\Delta F=-6.6 \mathrm{kcal} / \mathrm{mol}$, thus favoring the electron transfer towards the reaction center. The reorganization free energy is $\lambda=\sim 62 \mathrm{kcal} / \mathrm{mol}$ and the barrier height is $\Delta F^{*}=15 \mathrm{kcal} / \mathrm{mol}$. Both experimental ${ }^{10}$ and theorical ${ }^{7}$ studies have predicted a stable FS4C ${ }^{\text {red }}$. 

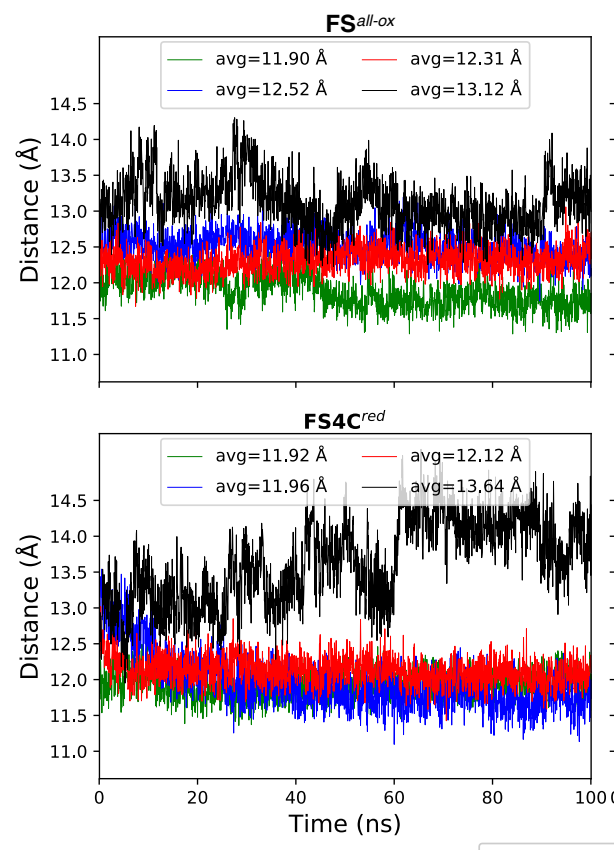

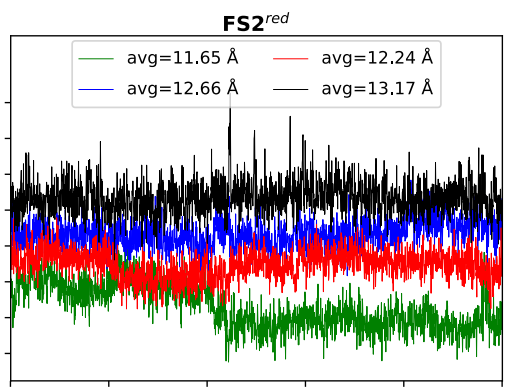

FS4B $^{\text {red }}$

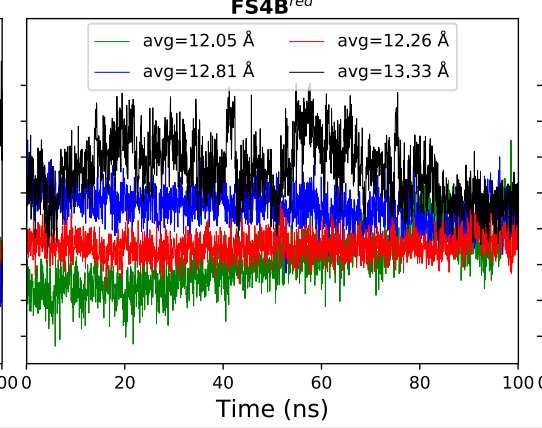

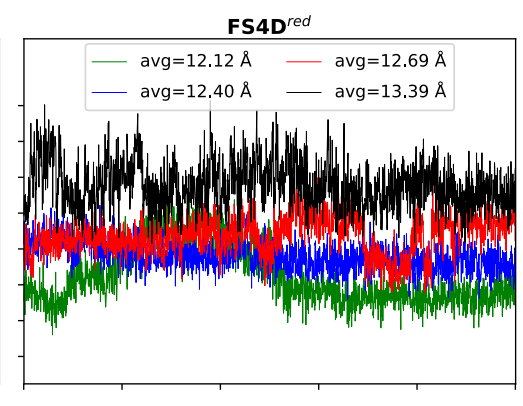

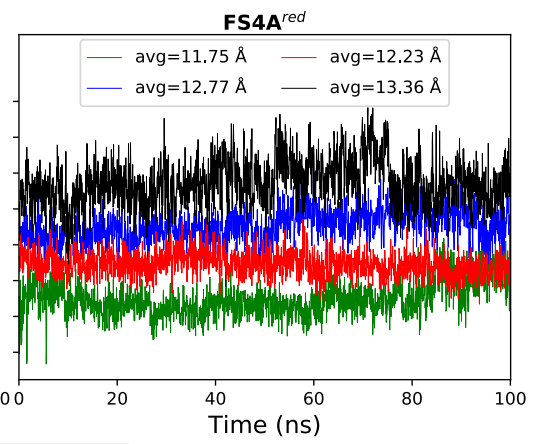

Figure 9: Distances between the centers of mass of the FeS clusters in the different oxidation states of the clusters. The average distance between the clusters are shown in the figure.

These numbers, which are also compiled in Table 1, are obtained directly from the graphs, rather than from equations 2, 3, and 4. The equations are derived under Marcus' linear response assumption, which implies that the two free energy curves are parabolic with equal curvature. From the graph, and also from the listed $\sigma$ values in the table, we see that the product curve is somewhat wider than the reactant curve, thus implying a deviation from Marcus' behavior. Therefore, inserting the measured average $\Delta E$ values, listed in Table 1 , into the equations for $\Delta F$ and $\lambda$ would result in slightly different numbers. The non-linear behavior is perhaps not unexpected now that we know the detailed molecular reorganization described above. The assumed Gaussian $\Delta E$ distribution in Marcus theory is due to the sum of many reorienting polar interactions in the protein and solvent environment, which act as random variables, each with their own distribution, as follows from the central limit theorem. However, in the hydrogenase system, we observe a rather specific rearrangement, the formation of a water channel in the protein leading to hydration of the cubane cluster; 
this can result in an additional non-linear contribution to the $\Delta E$ distribution. The deviation from ideal Marcus behavior was also reported previously for the [FeFe] hydrogenase enzyme. ${ }^{7}$

The free energy profile of the next step in the process is shown in Figure 10b. Electron transfer from FS4C to FS4B is more favorable with a reaction free energy of $-8 \mathrm{kcal} / \mathrm{mol}$ and an activation barrier of $9.6 \mathrm{kcal} / \mathrm{mol}$. The reorganization energy is $\sim 58 \mathrm{kcal} / \mathrm{mol}$. The final electron transfer step is from FS4B to FS4A. The Marcus plots show that the transfer between the last two clusters is almost energetically neutral with a $\Delta F$ of only $2 \mathrm{kcal} / \mathrm{mol}$ and a barrier of $13.7 \mathrm{kcal} / \mathrm{mol}$. The reorganization energy is, with $\sim 43 \mathrm{kcal} / \mathrm{mol}$, smaller than that of the other two electron transfer steps.

Experimentally the reduction potentials of the cubane cofactors embedded in the di-iron hydrogenase have been determined using potentiometric titrations in combination with electron paramagnetic resonance (EPR) spectroscopy. ${ }^{10}$ These show that the outer FS4D cluster is most difficult to reduce with a potential of $\leq 450 \mathrm{mV}$ with respect to the standard hydrogen electrode (SHE). The other potentials are FS4C: -368 mv, FS4B: -366 mV, and FS4A: -376 mV. We note that our computed transfer free energies are about an order of magnitude larger than the differences between these experimental reduction potentials. Previous MD simulation estimates of the (absolute) transfer free energies were even significantly larger (11.7, -14.8, -17.1 kcal/mol for transfer steps 1 to 3$).{ }^{7}$ There are several approximations in our model that could lead to discrepancies, including the classical point charge model to describe the cubane oxidation states, the limited statistics of sampling the protein and solvent reorganization, the finite size of our periodic simulation box, and the assumed Gaussian shape of the $\Delta E$ distributions. However, the effects of these approximations on the qualitative picture of the protein/solvent reorganization underlying the electron transfer mechanism in FeFe hydrogenase can be expected to be rather small. 

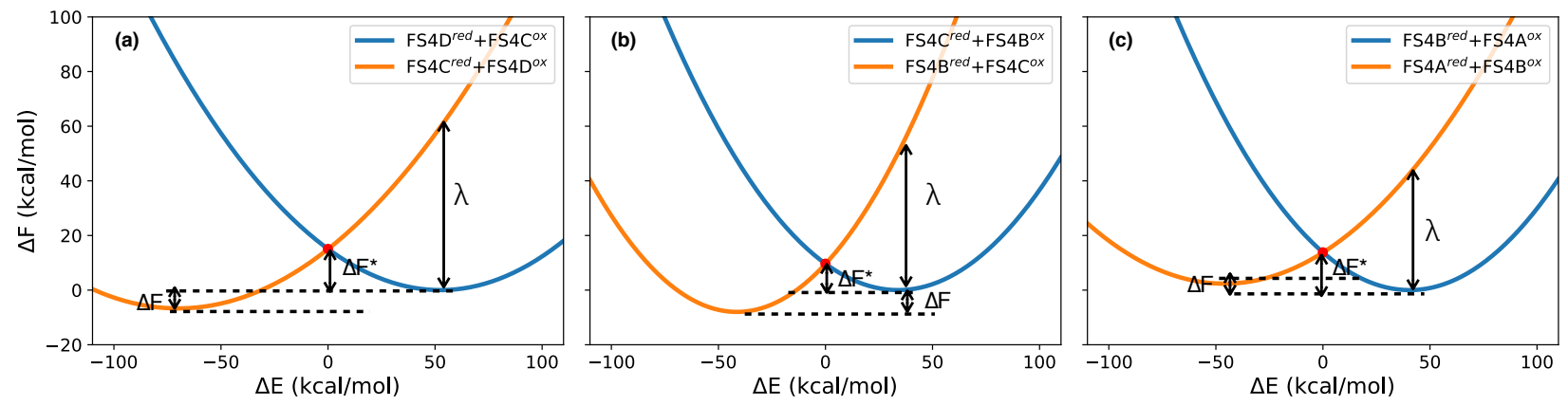

Figure 10: Marcus plots for the three electron transfer reactions: (a) FS4D $\rightarrow$ FS4C, (b) $\mathrm{FS} 4 \mathrm{C} \rightarrow \mathrm{FS} 4 \mathrm{~B}$, and (c) FS4B $\rightarrow \mathrm{FS} 4 \mathrm{~A}$. The electron transfer free energy $(\Delta F)$, the reorganization energy $(\lambda)$, and the activation free energy barrier $\left(\Delta F^{*}\right)$ are indicated and compiled in Table 1 . Note that the direction of the reactions on the $x$-axis is from right to left.

Table 1: Top: Averages and standard deviations of the computed $\Delta E$ distributions for the three electron transfer reactions. Transfer free energies, reorganization free energes and free energy barriers obtained from the free energy curves shown in Figure 10. All values are in $\mathrm{kcal} / \mathrm{mol}$.

\begin{tabular}{l|ccc}
\hline \hline & $\mathrm{FS} 4 \mathrm{D} \rightarrow \mathrm{FS} 4 \mathrm{C}$ & $\mathrm{FS} 4 \mathrm{C} \rightarrow \mathrm{FS} 4 \mathrm{~B}$ & $\mathrm{FS} 4 \mathrm{~B} \rightarrow \mathrm{FS} 4 \mathrm{~A}$ \\
\hline$\langle\Delta E\rangle_{R}$ & 52.5 & 33.9 & 40.5 \\
$\langle\Delta E\rangle_{P}$ & -70.1 & -41.4 & -45.9 \\
$\sigma_{R}$ & 7.4 & 5.9 & 6.0 \\
$\sigma_{P}$ & 8.2 & 5.3 & 7.2 \\
\hline$\Delta F$ & -6.6 & -8.0 & 2.0 \\
$\lambda$ & 62 & 58 & 43 \\
$\Delta F^{*}$ & 15 & 9.6 & 13.7 \\
\hline \hline
\end{tabular}

\section{Conclusions}

We have investigated the electron transport process in [FeFe] hydrogenase enzyme using classical molecular dynamics simulations. Electrons can be transported from the protein exterior to the reactive center via a series of four iron-sulfur cubane cofactors. The last cubane cluster 
is connected to the catalytic site where protons are reduced to form molecular hydrogen. We have simulated the enzyme in the oxidized resting state and in four reduced states, in which every time one of the cubane clusters has absorbed an electron. Our simulations show that the hydrogenase protein is very permeable to solvent water, allowing for the formation of water channels toward the cubane clusters. In particular, when a cubane cluster is reduced, a water channel is formed to that cluster and hydration of certain sulfur and iron atoms takes place. This substantial reorganization of the protein and solvent environment upon the transfer of an electron from one cubane cluster to the next stabilizes the oxidized and reduced states of the clusters. The amount of cubane hydration increases in a non-linear manner with the amount of charge transferred to the cluster, which is most likely the main cause of the significant deviations seen from the prototypical Marcus' behavior in the free energy profiles of the electron transfer reactions. The first two electron transfer steps are downhill by a few $\mathrm{kcal} / \mathrm{mol}$, whereas the last transfer step is uphill by $2 \mathrm{kcal} / \mathrm{mol}$. The largest barrier occurs at the first step and is $15 \mathrm{kcal} / \mathrm{mol}$. 


\section{References}

(1) Ferreira, K. N.; Iverson, T. M.; Maghlaoui, K.; Barber, J.; Iwata, S. Science 2004, 303, $1831-1838$.

(2) Hirst, J. Annu. Rev. Biochem. 2013, 82, 551-575.

(3) Martin, D. R.; Matyushov, D. V. Sci. Rep. 2017, 7, 5495.

(4) Fontecilla-Camps, J. C.; Volbeda, A.; Cavazza, C.; Nicolet, Y. Chem. Rev. 2007, 107, 4273-4303.

(5) Morra, S.; Giraudo, A.; Di Nardo, G.; King, P. W.; Gilardi, G.; Valetti, F. PloS one 2012, 7, e48400.

(6) Skourtis, S. S.; Waldeck, D. H.; Beratan, D. N. Annu. Rev. Phys. Chem. 2010, 61, $461-485$.

(7) McCullagh, M.; Voth, G. A. J. Phys. Chem. B 2013, 117, 4062-4071.

(8) Parey, K.; Wirth, C.; Vonck, J.; Zickermann, V. Curr. Opin. Struct. Biol. 2020, 63, 1 $-9$.

(9) Yoga, E. G.; Angerer, H.; Parey, K.; Zickermann, V. BBA - Bioenerg. 2020, 1861, 148153.

(10) Artz, J. H.; Mulder, D. W.; Ratzloff, M. W.; Lubner, C. E.; Zadvornyy, O. A.; LeVan, A. X.; Williams, S. G.; Adams, M. W.; Jones, A. K.; King, P. W. J. Am. Chem. Soc. 2017, 139, 9544-9550.

(11) Peters, J. W.; Lanzilotta, W. N.; Lemon, B. J.; Seefeldt, L. C. Science 1998, 282, $1853-1858$. 
(12) Pronk, S.; Páll, S.; Schulz, R.; Larsson, P.; Bjelkmar, P.; Apostolov, R.; Shirts, M. R.; Smith, J. C.; Kasson, P. M.; van der Spoel, Bioinformatics 2013, 29, 845-854.

(13) MacKerell Jr, A. D.; Bashford, D.; Bellott, M.; Dunbrack Jr, R. L.; Evanseck, J. D.; Field, M. J.; Fischer, S.; Gao, J.; Guo, H.; Ha, S. J. Phys. Chem. B 1998, 102, 35863616.

(14) MacKerell Jr, A. D.; Feig, M.; Brooks, C. L. J. Am. Chem. Soc. 2004, 126, 698-699.

(15) Chang, C. H.; Kim, K. J. Chem. Theory Comput. 2009, 5, 1137-1145.

(16) Mark, P.; Nilsson, L. J. Phys. Chem. A 2001, 105, 9954-9960.

(17) Hess, B.; Bekker, H.; Berendsen, H. J.; Fraaije, J. G. J. Comput. Chem. 1997, 18, $1463-1472$.

(18) Bussi, G.; Donadio, D.; Parrinello, M. J. Chem. Phys. 2007, 126, 014101.

(19) Parrinello, M.; Rahman, A. J. Phys. Colloq. 1981, 42, C6-511.

(20) Marcus, R. A.; Sutin, N. BBA-Rev. Bioenerg. 1985, 811, 265-322.

(21) Small, D. W.; Matyushov, D. V.; Voth, G. A. J. Am. Chem. Soc. 2003, 125, 7470-7478.

(22) Blumberger, J. Chem. Rev. 2015, 115, 11191-11238.

(23) Blumberger, J. Phys. Chem. Chem. Phys. 2008, 10, 5651-5667.

(24) Kilic, M.; Ensing, B. J. Phys. Chem. B 2019, 123, 9751-9761.

(25) Humphrey, W.; Dalke, A.; Schulten, K. J. Mol. Graphics 1996, 14, 33-38.

(26) Rodriguez-Macia, P.; Pawlak, K.; Rudiger, O.; Reijerse, E. J.; Lubitz, W.; Birrell, J. A. J. Am. Chem. Soc. 2017, 139, 15122-15134. 
(27) Hayashi, T.; Stuchebrukhov, A. A. Proc. Natl. Acad. Sci. 2010, 107, 19157-19162.

(28) Hayashi, T.; Stuchebrukhov, A. A. J. Phys.Chem. B 2011, 115, 5354-5364.

(29) Kaila, V. R.; Wikström, M.; Hummer, G. Proc. Natl. Acad. Sci. 2014, 111, 6988-6993.

(30) Sharma, V.; Belevich, G.; Gamiz-Hernandez, A. P.; Róg, T.; Vattulainen, I.; Verkhovskaya, M. L.; Wikström, M.; Hummer, G.; Kaila, V. R. Proc. Natl. Acad. Sci. 2015, 112, 11571-11576.

(31) Kaila, V. R. J. R. Soc., Interface 2018, 15, 20170916. 